\title{
PARTICLE FLOW FOR SEQUENTIAL MONTE CARLO IMPLEMENTATION OF PROBABILITY HYPOTHESIS DENSITY
}

\author{
Yang Liu ${ }^{1}$, Wenwu Wang ${ }^{1}$, Yuxin Zhao ${ }^{2}$ \\ ${ }^{1}$ Department of Electrical and Electronic Engineering, University of Surrey, UK \\ ${ }^{2}$ College of Automation, Harbin Engineering University, China
}

\begin{abstract}
Target tracking is a challenging task and generally no analytical solution is available, especially for the multi-target tracking systems. To address this problem, probability hypothesis density (PHD) filter is used by propagating the PHD instead of the full multi-target posterior. Recently, the particle flow filter based on the log homotopy provides a new way for state estimation. In this paper, we propose a novel sequential Monte Carlo (SMC) implementation for the PHD filter assisted by the particle flow (PF), which is called PF-SMCPHD filter. Experimental results show that our proposed filter has higher accuracy than the SMC-PHD filter and is computationally cheaper than the Gaussian mixture PHD (GM-PHD) filter.
\end{abstract}

Index Terms - tracking system, SMC-PHD, particle flow, multi-target

\section{INTRODUCTION}

Multi-target tracking is a frequently occurring problem in which the number of targets varies over time. The observations used in multi-target tracking often include random errors, spurious returns from clutters, and detection loss [1]. Apart from that, there are other challenges, for example, 1) the number of targets is unknown and changing; 2) targets may be occluded. To address these problems, Mahler proposed to approximate the multi-object Bayesian recursion by propagating the probability hypothesis density (PHD) [2], which does not involve data association due to the use of the random finite set (RFS) [3] framework. The PHD filter has been implemented as the sequential Monte Carlo (SMCPHD) filter [4], the Gaussian mixture PHD (GM-PHD) filter [5] and the cardinalized PHD (CPHD) filter [6]. To solve the non-Gaussian and non-linear problem, the standard SMC implementation of the PHD filter is often used which, however, entails a large set of weighted particles [7]. The main

\footnotetext{
This work was supported by the EPSRC Programme Grant S3A: Future Spatial Audio for an Immersive Listener Experience at Home (EP/L000539/1), the BBC as part of the BBC Audio Research Partnership, the China Scholarship Council (CSC) and the EPSRC grant EP/K014307/1 and the MOD University Defence Research Collaboration in Signal Processing.
}

disadvantage of SMC-PHD filter is the weight degeneracy problem. After some updates, only few particles have significant weights. The prior distribution does not overlap with the target distribution [8].

As a different algorithm for solving the non-linear and non-Gaussian problem, Duam and Huang introduced a particle flow filter $[9,10,11,12]$. The key idea is to migrate particles from the unnormalized prior density to the posterior density by a physical flow. Recently, particle flow has been used to improve the accuracy of the particle filter [13], called the particle flow particle filter (PFPF). The particle cloud retains a higher effective sample size (ESS) [14]. However, for multi-target tracking, a dependent filter needs to be applied to each target, which introduces the problem of model-data association [15]. To solve this problem, Zhao et al. [16] proposed a Gaussian mixture particle flow PHD filter, called GPF-PHD filter. However, in this filter, the particles are generated for each target, and the computational cost could be high for a large number of targets and clutters. Due to the use of the Gaussian Mixture model, this method is less robust than the SMC-PHD filter for non-linear and non-Gaussian problems.

In this paper, we use particle flow to mitigate the weight degeneracy problem and thereby to improve the accuracy of the SMC-PHD filter. This new filter is called the PF-SMCPHD filter. More specifically, we use particle flow to adjust the states and weights of the particles based on the observations. The number and positions of the targets are assumed unknown. Therefore the number of flows created is determined based on the number of observations. The computational complexity for generating each flow is controlled by choosing the most relevant particles from the whole particle set, and the assignment of the ambiguous particles, i.e. those having a similar distance to two (or more) different observations, to each flow is assisted by particle duplication.

\section{SMC-PHD FILTER AND PARTICLE FLOW}

We assume that the target dynamics and observations are described as a Markov state-space signal model:

$$
\begin{gathered}
\widetilde{\boldsymbol{m}}_{k}=\boldsymbol{f}_{\widetilde{\boldsymbol{m}}}\left(\widetilde{\boldsymbol{m}}_{k-1}, \boldsymbol{\tau}_{k}\right), \\
\boldsymbol{z}_{k}=\boldsymbol{f}_{\boldsymbol{z}}\left(\widetilde{\boldsymbol{m}}_{k}, \varsigma_{k}\right)
\end{gathered}
$$


where $\widetilde{\boldsymbol{m}}_{k} \in \mathbb{R}^{d_{m}}$ is the target state vector at time-step $k$, is used to distinguish the target state from the particle state used later, and $\boldsymbol{z}_{k} \in \mathbb{R}^{d_{z}}$ is the observation vector. In this paper, the state $\widetilde{\boldsymbol{m}}_{k}=\left[x_{k}, y_{k}, \dot{x}_{k}, \dot{y}_{k}\right]^{T}$ consists of positions $\left(x_{k}, y_{k}\right)$ and velocities $\left(\dot{x}_{k}, \dot{y}_{k}\right)$, while the observation is a noisy version of the position. So $d_{m}=4$ and $d_{z}=2$. $\tau_{k}$ and $\varsigma_{k}$ are system excitation and observation noise terms, respectively. $\boldsymbol{f}_{\widetilde{\boldsymbol{m}}}$ is the transition density and $\boldsymbol{f}_{\boldsymbol{z}}$ is the nonlinear observation model.

\subsection{SMC-PHD filter}

The PHD is the first-order moment of the RFS, which is a set of random variables (or vectors) with the random cardinality. Suppose that at time step $k-1$, we have a set of $N$ particles $\left\{\boldsymbol{m}_{k-1}^{i}\right\}_{i=1}^{N}$ and weights $\left\{\omega_{k-1}^{i}\right\}_{i=1}^{N}$, which represent the posterior distribution at time $k-1$.

For the SMC implementation of the prediction step [4], the particle set is obtained by the proposal distribution $q_{k}\left(\boldsymbol{m}_{k \mid k-1}^{i} \mid \boldsymbol{m}_{k-1}^{i}, \boldsymbol{Z}_{k}\right)$, where $\boldsymbol{Z}_{k}$ is the set of observations,

$$
\boldsymbol{m}_{k \mid k-1}^{i} \sim q_{k}\left(\cdot \mid \boldsymbol{m}_{k-1}^{i}, \boldsymbol{Z}_{k}\right) .
$$

Their weights are

$\omega_{k \mid k-1}^{i}=\frac{\phi_{k \mid k-1}\left(\boldsymbol{m}_{k \mid k-1}^{i} \mid \boldsymbol{m}_{k-1}^{i}\right) \omega_{k-1}^{i}}{q_{k}\left(\boldsymbol{m}_{k \mid k-1}^{i} \mid \boldsymbol{m}_{k-1}^{i}, \boldsymbol{Z}_{k}\right)} \quad i=1, \ldots, N$

where $\phi_{k \mid k-1}\left(\boldsymbol{m}_{k \mid k-1}^{i} \mid \boldsymbol{m}_{k-1}^{i}\right)$ is the analogue of the state transition probability with the previous state $\boldsymbol{m}_{k-1}^{i} . N_{\Gamma}$ particles are sampled from the new born importance function $p_{k}\left(\boldsymbol{m}_{k \mid k-1}^{i} \mid \boldsymbol{Z}_{k}\right)$

$$
\boldsymbol{m}_{k \mid k-1}^{i} \sim p_{k}\left(\cdot \mid \boldsymbol{Z}_{k}\right)
$$

Their weights are

$$
\omega_{k \mid k-1}^{i}=\frac{\gamma_{k}\left(\boldsymbol{m}_{k \mid k-1}^{i}\right)}{N_{\Gamma} p_{k}\left(\boldsymbol{m}_{k \mid k-1}^{i} \mid \boldsymbol{Z}_{k}\right)} \quad i=N+1, \ldots, N+N_{\Gamma} .
$$

In the update step, the weights are calculated as

$$
\omega_{k}^{i}=\left[1-p_{D, k}\left(\boldsymbol{m}_{k}^{i}\right)+\sum_{\boldsymbol{z} \in \boldsymbol{Z}_{k}} \frac{p_{D, k}\left(\boldsymbol{m}_{k}^{i}\right) g_{k}\left(\boldsymbol{z} \mid \boldsymbol{m}_{k}^{i}\right)}{\kappa_{k}(\boldsymbol{z})+C_{k}(\boldsymbol{z})}\right] \omega_{k \mid k-1}^{i}
$$

where

$$
C_{k}(\boldsymbol{z})=\sum_{i=1}^{N+N_{\Gamma}} p_{D, k}\left(\boldsymbol{m}_{k}^{i}\right) g_{k}\left(\boldsymbol{z} \mid \boldsymbol{m}_{k}^{i}\right) \omega_{k \mid k-1}^{i}
$$

in which $\kappa_{k}(\boldsymbol{z})$ denotes the clutter intensity of the observation $z$ at time $k . p_{D, k}$ is the probability of detection at time $k$. $g_{k}(. \mid$.$) is the likelihood of individual targets.$

The number of targets is estimated by the sum of weights,

$$
\widetilde{N}=\sum_{i=1}^{N+N_{\Gamma}} \omega_{k}^{i}
$$

The states and the weights of the targets $\left\{\widetilde{\boldsymbol{m}}_{k}^{j}, \widetilde{\omega}_{k}^{j}\right\}_{j=1}^{\widetilde{N}}$ can then be calculated with e.g. the k-means clustering method [17] or the Multi-expected a Posterior (MEAP) [18, 19].

Finally, we perform re-sampling when the ESS [14] is smaller than half of the total number of particles. In the re-sampling step, we can obtain $\left\{\boldsymbol{m}_{k}^{i}, \omega_{k}^{i}\right\}_{i=1}^{N}$, where $\left\{\omega_{k}^{i}\right\}_{i=1}^{N}=1 / N$. The pseudo-code of the SMC-PHD filter is summarized in Algorithm 1.

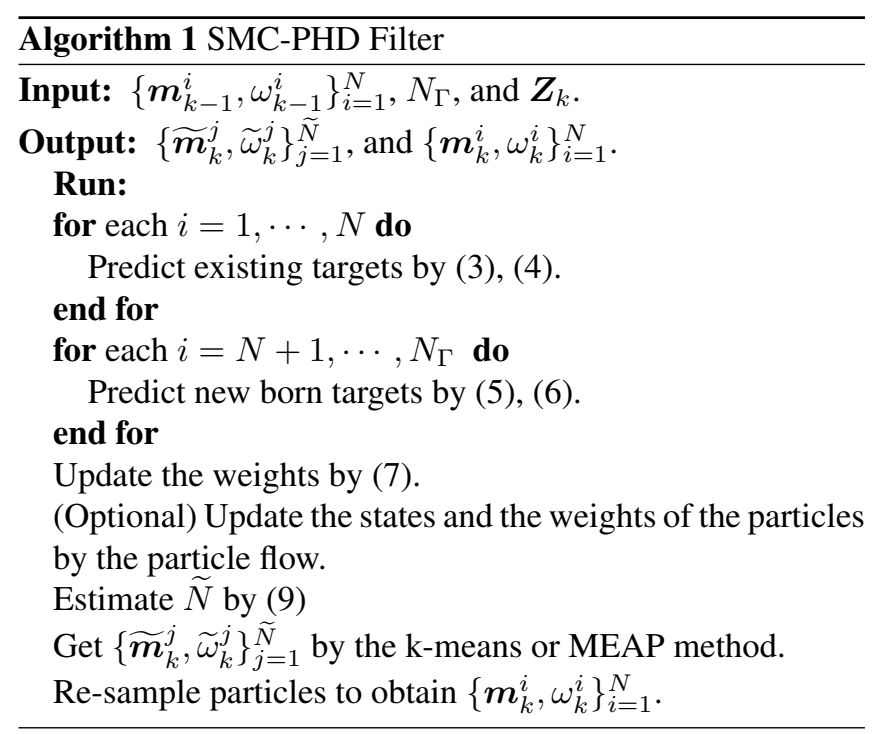

\subsection{Particle flow}

A homotopy function can be defined to model the particle flow process [20],

$$
\log \left(\psi_{k}(\boldsymbol{m}, \lambda)\right)=\log \left(h_{k}(\boldsymbol{m})\right)+\lambda \log \left(g_{k}(\boldsymbol{m})\right)
$$

where $\lambda$ is a step size parameter taking values from the set $\left[0, \triangle \lambda, 2 \triangle \lambda, \cdots, N_{\lambda} \triangle \lambda\right]$ and $N_{\lambda} \triangle \lambda=1$. When $\lambda=0$, $\psi_{k}$ represents the prior density $h$. With $\lambda$ varying to $1, \psi_{k}$ is translated into the normalized posterior density [10]. When the prior and the likelihood are unnormalized Gaussian probability densities, the exact solution for the particle flow is:

$$
\frac{d \boldsymbol{m}}{d \lambda}=\boldsymbol{A}(\lambda) \boldsymbol{m}+\boldsymbol{b}(\lambda)
$$

where

$$
\begin{aligned}
\boldsymbol{A}(\lambda) & =-\frac{1}{2} \boldsymbol{P} \boldsymbol{H}^{T}\left(\lambda \boldsymbol{H} \boldsymbol{P} \boldsymbol{H}^{T}+\boldsymbol{R}\right)^{-1} \boldsymbol{H} \\
\boldsymbol{b}(\lambda) & =(\boldsymbol{I}+2 \lambda \boldsymbol{A})\left[(\boldsymbol{I}+\lambda \boldsymbol{A}) \boldsymbol{P} \boldsymbol{H}^{T} \boldsymbol{R}^{-1} \boldsymbol{z}+\boldsymbol{A} \overline{\boldsymbol{m}}\right]
\end{aligned}
$$

in which $\overline{\boldsymbol{m}}$ is the mean of the particles. $\boldsymbol{R}$ is the covariance matrix of the observation noise, which is given before running the algorithm. $\boldsymbol{P}$ is the covariance matrix of the particles. $\boldsymbol{H}$ is computed as the Jacobian matrix,

$$
\boldsymbol{H}=\left[\begin{array}{cc}
\cos (\theta) & -\sin (\theta) \\
\sin (\theta) & \cos (\theta)
\end{array}\right]
$$


where $\theta=\arctan \left(\frac{\boldsymbol{m}(2)}{\boldsymbol{m}(1)}\right)$, and $\boldsymbol{m}(1)$ and $\boldsymbol{m}(2)$ are the first and second element of $\boldsymbol{m}$, respectively.

\section{PROPOSED PARTICLE FLOW SMC-PHD FILTER}

In conventional SMC-PHD filter as presented in Algorithm 1, particles are drawn from a proposal distribution, which may not be well matched to the posterior density. To mitigate this problem, we add an adjustment step between the prediction step and update step, where the particle flow is incorporated to adjust the states and weights of the particles by smoothly migrating them from the prior to the posterior density.

In SMC-PHD, the prior information about the number of targets and the association between the targets and particles is unknown and varying. To solve this problem, we create the particle flows based on the observations. More specifically, we generate the same number of flows as that of the observations. Nevertheless, the computational cost associated with the evaluation of Eqs. (11)-(14) could be high with the increase in the number of particles used for creating the flow. Therefore, we assume that each flow will only be influenced by the particles in the neighborhood of the corresponding observation within a certain distance $d$ to the observation. We choose particles $\left\{\boldsymbol{m}_{k \mid k-1}^{i}, \omega_{k \mid k-1}^{i}\right\}_{i \in \boldsymbol{\Lambda}(\boldsymbol{z})}$, where $\boldsymbol{\Lambda}(\boldsymbol{z})$ is a subset of $\boldsymbol{E}=\left[1, \cdots, N+N_{\Gamma}\right]$ determined based on the observation $\boldsymbol{z}$, defined as $\boldsymbol{\Lambda}(\boldsymbol{z})=\left\{i|| \boldsymbol{z}-\boldsymbol{m}_{k \mid k-1}^{i} \mid \leq d, i \in \boldsymbol{E}\right\}$, whose size is $N_{r}(\boldsymbol{z})$. Then, we calculate the mean $\overline{\boldsymbol{m}}(\boldsymbol{z})$ and covariance $\boldsymbol{P}(\boldsymbol{z})$ of these selected particles.

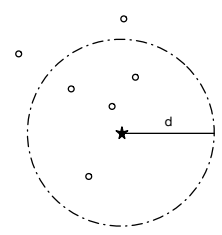

(a)

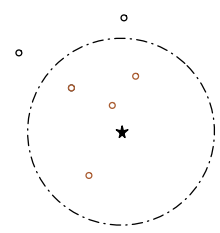

(b)

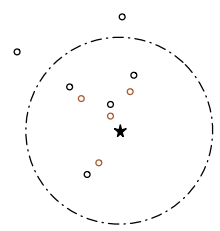

(c)
Fig. 1. The adjustment step in PF-SMC-PHD. The star represents an observation. The small solid circles denote particles. (a) shows the selected particles (within the dot-dashed circle). (b) shows the duplicated particles (in red). (c) shows the duplicated particles adjusted by the particle flow.

In practice, one particle may be in a similar distance to two (or more) different observations. This makes it difficult to assign such particle to an appropriate particle flow. To mitigate this issue, we duplicate the set of the selected particles, and create the flow using only the duplicated particles. Figure 1 shows this process for adjusting these particles, where the small solid circles represent the particles, the star shows the position of the observation $z$ and the dot-dashed circle with the radius $d$ shows the neighborhood area of $z$ from which the particles are selected (Figure 1(a)), with $\boldsymbol{\Lambda}(\boldsymbol{z})$ recording their indices. In Figure 1(b), the particles in the dot-dashed circle are duplicated (hence shown in red) which overlap with the selected particles (not shown). Figure 1(c) shows the result of the adjustment step where the duplicated particles are migrated by the particle flow. It is worth noting that, different from just randomly adding more particles, the states of observations and the information of the targets are better exploited by the proposed method for evaluating the mean and covariance matrix of the duplicated particles. The states of particles are updated by particle flow as Eq. (11) and their weights are updated for each observation:

$$
\omega_{k \mid k-1}^{i}=\frac{q_{k}\left(\boldsymbol{m}_{k \mid k-1}^{i} \mid \boldsymbol{m}_{k-1}^{i}, \boldsymbol{z}\right)}{q_{k}\left(\boldsymbol{m}_{k \mid k-1}^{i} \mid \boldsymbol{m}_{k-1}^{i}, \boldsymbol{z}\right)} \omega_{k \mid k-1}^{i}
$$

The new particle set is the union of the duplicated set and the original particle set. The pseudo-code of the adjustment step of PF-SMC-PHD is presented in Algorithm 2. Note that $\boldsymbol{m}$ in Eq. (11) is represented with $\boldsymbol{m}_{k \mid k-1}^{i}$. As the result of introducing the duplicated and new born particles, the total number of particles has been increased at the end of each iteration. However, as observed in our experiments, the ESS has been improved, i.e. less number of particles need to be resampled due to the use of the duplicated particles.

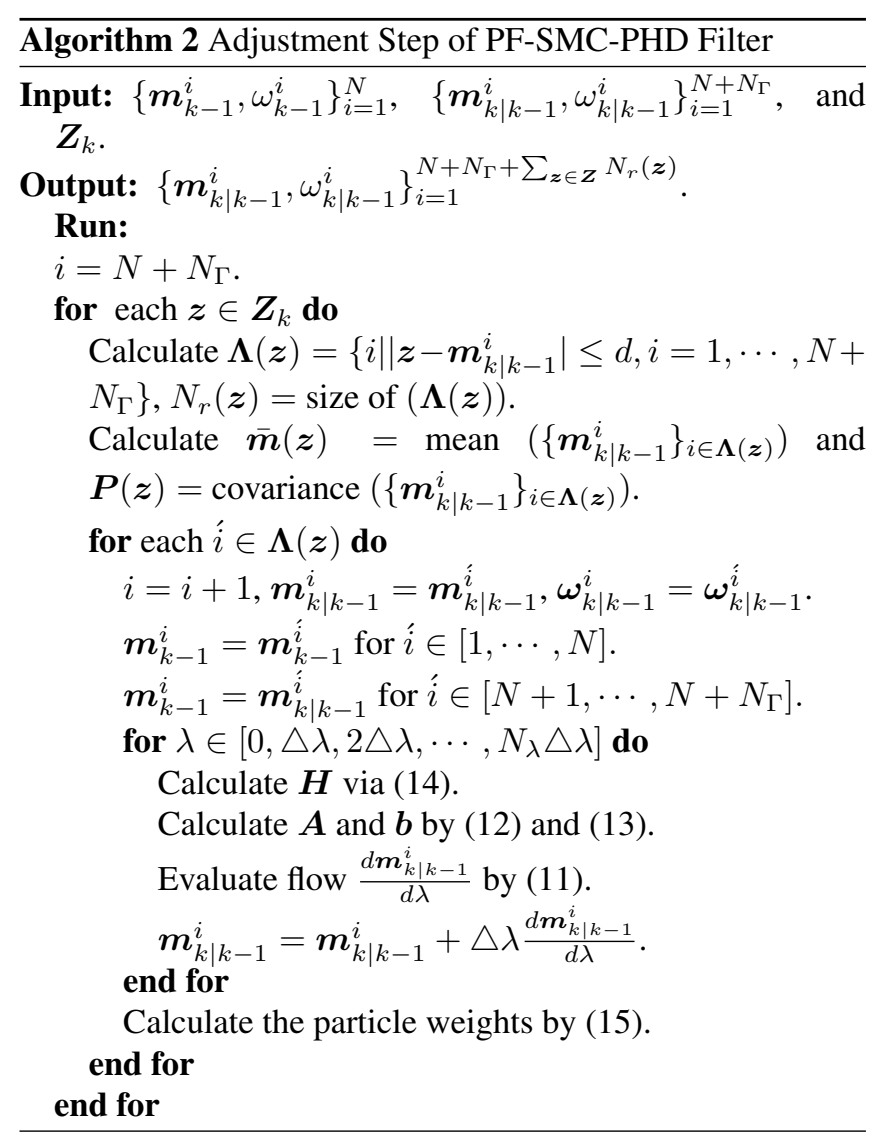




\section{SIMULATION EXPERIMENTS}

We designed an experiment for evaluating our novel PFSMC-PHD filter, as compared with the GM-PHD and the SMC-PHD filters, on a PC with Intel i7 $3.40 \mathrm{GHz}$ CPU and 8G RAM. We run our algorithm in Matlab on Windows 7.

In order to simulate the movements of the targets, we design an occlusion scenario, in which three targets move in the certain area $[-1000,1000] *[-1000,1000]$. The probability of detection $p_{D, k}=0.98$. There are 50 random clutters in observations and their positions are set randomly. The initial states of two targets are $\widetilde{\boldsymbol{m}}_{0}^{1}=[250,250,2.5,-11.5]^{T}$ and $\widetilde{\boldsymbol{m}}_{0}^{2}=[-250,-250,11.5,-2.5]^{T}$. The third target appears from $k=70$ and its initial state is $\widetilde{\boldsymbol{m}}_{0}^{3}=$ $[0,-400,30,-27]^{T}$.

The dynamic model is $\boldsymbol{f}_{\widetilde{\boldsymbol{m}}}=\left[\begin{array}{ll}\boldsymbol{I}_{2} & \boldsymbol{I}_{2} \\ \mathbf{0}_{2} & \boldsymbol{I}_{2}\end{array}\right]$ where $\boldsymbol{I}_{2}$ and $\mathbf{0}_{2}$ denote the $2 \times 2$ identity and zero matrices. $\boldsymbol{R}$ is set as $\left[\begin{array}{cc}25 \boldsymbol{I}_{2} & \mathbf{0}_{2} \\ \mathbf{0}_{2} & 25 \boldsymbol{I}_{2}\end{array}\right]$. The parameters of the GM-PHD filter are set as in [5]. In the SMC-PHD filter, there are 500 particles for each expected target. The parameters for the birth step of the SMC-PHD filter are the same as those for the GM-PHD filter. For particle flow of the proposed PF-SMC-PHD filter and $\boldsymbol{P}=[5,5,1,1]$. The distance $d$ is set as 30 . We test a set of $\triangle \lambda$ decreasing from 0.5 to 0.001 . Both the tracking error and the number of times required for re-sampling decrease gradually with the decrease in $\triangle \lambda$ until it reaches 0.01 . However, when $\triangle \lambda<0.01$, the improvement is negligible, but the computational load becomes very high. Due to page limit, we only show part of the results with $\triangle \lambda=0.01$.

Figure 2 shows the average position errors by the optimal sub-pattern assignment (OSPA) [15] at each time step. At $k=5,6,10$, the distance between the two targets is large and OPSA of the PF-SMC-PHD filter is only $20 \%$ of that of the SMC-PHD filter. After the third target appears, at $k=78$, the PF-SMC-PHD filter also gives lower OPSA than the SMCPHD filter. In Table 1, it can be observed that the k-means and MEAP give similar results. Although the computational cost of the PF-SMC-PHD filter is approximately 8 times that of the SMC-PHD filter, its OPSA error is reduced to more than half of that. As the number of particles remains fixed in this simulation, change in the number of targets would not affect the computational cost, but may lead to an increase in the OSPA error.

Figure 3 compares the average ESS [14] between the SMC-PHD and PF-PHD-SMC filters. Since k-means and MEAP give similar results as shown above, only the k-means clustering is used here. When $\log _{\frac{N}{2}}(E S S)<1$, the particles are degenerated and need to be re-sampled. For the whole process, the SMC-PHD filter re-samples the particles 12 times while PF-SMC-PHD filter re-samples them only 5 times. However, after the third target appearing at $k=70$, the re-sampling is used more frequently in both methods. This

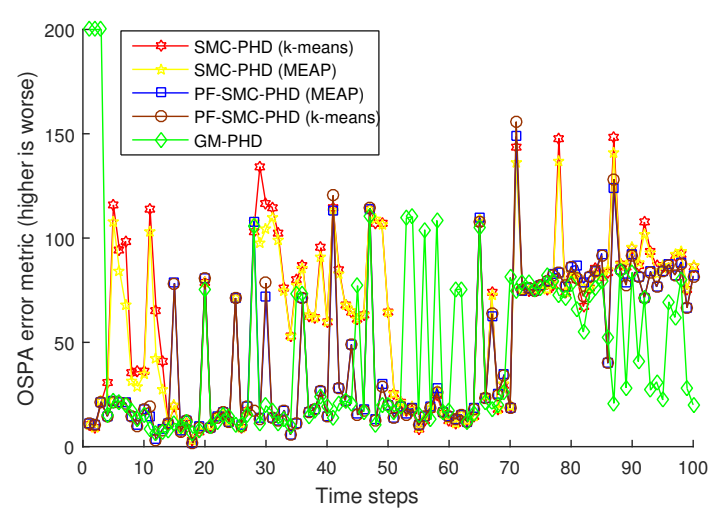

Fig. 2. Tracking performance of the compared filtering algorithms at each time step.

\begin{tabular}{|c|c|c|}
\hline algorithm & time(s) & average OSPA \\
\hline SMC-PHD with k-means & 6.7710 & 61.7306 \\
SMC-PHD with MEAP & 6.7680 & 59.8458 \\
PF-SMC-PHD with k-means & 51.5625 & 24.7594 \\
PF-SMC-PHD with MEAP & 51.5527 & 25.8386 \\
GM-PHD & 226 & 45.2033 \\
\hline
\end{tabular}

Table 1. Computing time and tracking OSPA of the compared filtering algorithms at each time step.

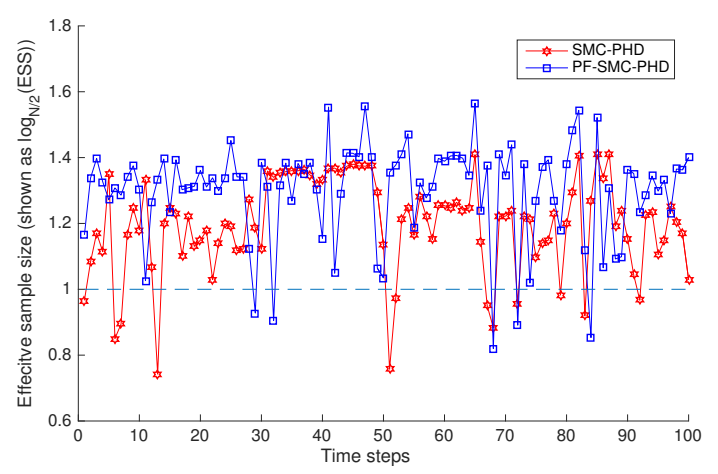

Fig. 3. ESS for SMC-PHD filter and PF-SMC-PHD filter.

implies that the weight degeneracy problem is worsened as a result of the appearance of a new target.

\section{CONCLUSION}

We have presented a novel particle flow SMC-PHD filter for multi-target tracking, by adding an adjustment step using particle flow to smoothly migrate the particles from the prior distribution to the posterior density. The proposed technique has been shown to improve the effective sample size and to mitigate the weight degeneracy problem, thus offering improved tracking accuracy over the SMC-PHD filter. 


\section{REFERENCES}

[1] V. Kılıç, M. Barnard, W. Wang, and J. Kittler, "Audio assisted robust visual tracking with adaptive particle filtering," IEEE Transactions on Multimedia, vol. 17, no. 2, pp. 186-200, 2015.

[2] R. P. Mahler, "Multitarget Bayes filtering via first-order multitarget moments," IEEE Transactions on Aerospace and Electronic Systems, vol. 39, no. 4, pp. 1152-1178, 2003.

[3] B.-N. Vo, S. Singh, and A. Doucet, "Sequential Monte Carlo implementation of the PHD filter for multi-target tracking," in Proc. Intl Conf. on Information Fusion, 2003, pp. 792-799.

[4] B. Ristic, D. Clark, and B.-N. Vo, "Improved SMC implementation of the PHD filter," in 13th Conference on Information Fusion. IEEE, 2010, pp. 1-8.

[5] B.-N. Vo and M. Wing-Kin, "The Gaussian mixture probability hypothesis density filter," IEEE Transactions on Signal Processing, vol. 54, no. 11, pp. 40914104, 2006.

[6] R. Mahler, "A theory of PHD filters of higher order in target number," in Defense and Security Symposium. International Society for Optics and Photonics, 2006, pp. 62350K-1-62350K-12.

[7] V. Kılıç, M. Barnard, W. Wang, A. Hilton, and J. Kittler, "Mean-shift and sparse sampling based SMC-PHD filtering for audio informed visual speaker tracking," IEEE Transactions on Multimedia, 2016.

[8] M. A. Khan and M. Ulmke, "Non-linear and nonGaussian state estimation using log-homotopy based particle flow filters," Workshop on Sensor Data Fusion: Trends, Solutions, Applications, 2014.

[9] F. Daum and J. Huang, "Particle flow for nonlinear filters," in 2011 IEEE International Conference on Acoustics, Speech and Signal Processing. IEEE, 2011, pp. 5920-5923.

[10] F. Daum and J. Huang, "Particle flow for nonlinear filters with log-homotopy," in SPIE Defense and Security Symposium. International Society for Optics and Photonics, 2008, pp. 696918-6969180.

[11] F. Daum and J. Huang, "Renormalization group flow and other ideas inspired by physics for nonlinear filters, Bayesian decisions, and transport," in SPIE Defense+ Security. International Society for Optics and Photonics, 2014, pp. 90910I-1-90910I-14.
[12] F. Daum and J. Huang, "Small curvature particle flow for nonlinear filters," in SPIE Defense, Security, and Sensing. International Society for Optics and Photonics, 2012, pp. 83930A-1-83930A-12.

[13] Y. Li, L. Zhao, and M. Coates, "Particle flow for particle filtering," in IEEE International Conference on Acoustics, Speech and Signal Processing. IEEE, 2016, pp. 3979-3983.

[14] A. Kong, J. S. Liu, and W. H. Wong, "Sequential imputations and Bayesian missing data problems," Journal of the American statistical association, vol. 89, no. 425, pp. 278-288, 1994.

[15] T. Fortmann, Y. Bar-Shalom, and M. Scheffe, "Sonar tracking of multiple targets using joint probabilistic data association," IEEE Journal of Oceanic Engineering, vol. 8, no. 3, pp. 173-184, 1983.

[16] L. Zhao, J. Wang, Y. Li, and M. J. Coates, "Gaussian particle flow implementation of PHD filter," in SPIE Defense + Security. International Society for Optics and Photonics, 2016, pp. 98420D-1-98420D-10.

[17] D. Arthur and S. Vassilvitskii, "k-means++: The advantages of careful seeding," in Proceedings of the 18th Annual ACM-SIAM Symposium on Discrete Algorithms. Society for Industrial and Applied Mathematics, 2007, pp. 1027-1035.

[18] T. Li, S. Sun, M. Bolić, and J. M. Corchado, "Algorithm design for parallel implementation of the SMC-PHD filter,' Signal Processing, vol. 119, pp. 115-127, 2016.

[19] T. Li, S. Sun, J. M. Corchado, and M. F. Siyau, "A particle dyeing approach for track continuity for the SMCPHD filter," in Information Fusion (FUSION), 2014 17th International Conference on. IEEE, 2014, pp. 18.

[20] F. Daum, J. Huang, and A. Noushin, "Exact particle flow for nonlinear filters," in SPIE Defense, Security, and Sensing. International society for optics and photonics, 2010, pp. 769704-1-769704-19. 